\title{
Water-Based Metasurfaces for Effective Switching of Microwaves
}

\author{
Jacobsen, Rasmus Elkjær; Lavrinenko, Andrei V.; Arslanagic, Samel
}

Published in:

I E E E Antennas and Wireless Propagation Letters

Link to article, DOI:

10.1109/LAWP.2018.2803214

Publication date:

2018

Document Version

Peer reviewed version

Link back to DTU Orbit

Citation (APA):

Jacobsen, R. E., Lavrinenko, A. V., \& Arslanagic, S. (2018). Water-Based Metasurfaces for Effective Switching of Microwaves. I E E E Antennas and Wireless Propagation Letters, 17(4), 571-574.

https://doi.org/10.1109/LAWP.2018.2803214

\section{General rights}

Copyright and moral rights for the publications made accessible in the public portal are retained by the authors and/or other copyright owners and it is a condition of accessing publications that users recognise and abide by the legal requirements associated with these rights.

- Users may download and print one copy of any publication from the public portal for the purpose of private study or research.

- You may not further distribute the material or use it for any profit-making activity or commercial gain

- You may freely distribute the URL identifying the publication in the public portal

If you believe that this document breaches copyright please contact us providing details, and we will remove access to the work immediately and investigate your claim 


\title{
Water-Based Metasurfaces for Effective Switching of Microwaves
}

\author{
Rasmus E. Jacobsen, Andrei V. Lavrinenko, and Samel Arslanagić, Member, IEEE
}

\begin{abstract}
All-dielectric metasurfaces have recently attracted great attention in the artificial material design and related applications. Among a variety of materials, water was recently proposed for strongly resonant inclusions in such configurations due to its relatively high permittivity. We presently design and characterize polarization sensitive metasurfaces with simple and easily fabricated "rod-like" water inclusions in a low permittivity host, which may be tuned mechanically by rotation. Excellent agreement is reported between simulations and the experimental results, obtained in an L-band waveguide environment. Moreover, we show effective guiding and s witching effects of the fields radiated by a dipole antenna located next to a stack of metasurfaces. The proposed metasurfaces may be very convenient for a variety of microwave applications owing to the low cost, abundance and bio-friendly nature of water.
\end{abstract}

Index Terms - metasurfaces, waters, resonances, microwaves

\section{INTRODUCTION}

A 1l-dielectric metamaterials (MMs) and metasurfaces (MSs) offer significant wave-manipulation advantages in comparison to metal-based artificial materials due to significantly lower material losses [1-3]. Their extraordinary properties rely on properly shaped high-permittivity inclusions situated in a low dielectric matrix. At radio and microwave frequencies a number of very high permittivity ceramics exist such as barium strontium titanate [4]. These materials are, however, often very costly and their dynamic properties are not easy to tune. A true technological breakthrough of alldielectric MMs and MSs requires simple and cheap solutions with low losses and dynamically tunable properties. To this end, water has been recently proposed as an interesting candidate for all-dielectric MM inclusions [5-7]. Although its losses might be a problem at some frequencies, its abundance, price and bio-friendly nature combined with the frequency and temperature dependent permittivity make it an interesting candidate in MM design. Moreover, due to its liquid form, it takes on the shape of any container. Consequently, tuning the properties of an array consisting of water-based inclusions can be done thermally, mechanically and even by gravitation [5]. The first experimental demonstration of a mechanically tuned

R. E. Jacobsen and A. V. Lavrinenko are with the Department of Photonics Engineering, Technical University of Denmark, Bld. 345A, Ørsteds Plads, 2800 Kgs. Lyngby, Denmark (e-mail: alav@fotonik.dtu.dk).

S. Arslanagic is with the Department of Electrical Engineering, Technical University of Denmark, Bld. 348, Ørsteds Plads, 2800 Kgs. Lyngby, Denmark (e-mail: sar@elektro.dtu.dk).
MS with inclusions partially filled with water was reported in [8], but the performance was not optimal. A thermally tunable water-based MM, capable of switching between elliptic and hyperbolic regimes was reported in [9]. A metallic MS on a water-filled substrate as a broadband thermally tunable absorber was presented in [10]. A giant field enhancement was directly measured in water-based cylinders [11], and reconfigurable all-dielectric MSs with tunable chemical systems in aqueous solution were recently demonstrated [12], further cementing the great potential of water for an extended variety of applications.

In this work, we conduct modeling and characterization of water-based polarization-sensitive MSs with simple and easily fabricated "rod-like" water elements in a dielectric Rohacell $51 \mathrm{HF}$ matrix. Excellent agreement between numerical and experimental results is demonstrated. Switching of the MS from the transmission to reflection mode is achieved with a simple mechanical rotation by $90^{\circ}$. Such behavior can be enhanced by stacking of MSs in free space. Specifically, we show how stacked MSs, for one orientation of the "rod-like" elements, can guide waves emitted by a nearby dipole antenna, while their rotation by $90^{\circ}$ effectively switches off the radiation in the desired direction. The proposed water-based MSs may serve as cheap and bio-friendly microwave sensors for identification and characterization of various water plastic contaminants, and as prototypes of optical and terahertz structures. Tunability of such devices is granted by strong dependence of water properties on temperature and frequency, which can be supported by mechanical reshaping of flexible containers or their partial infiltration with water.

The paper is organized as follows. Section II introduces the MS with water unit cells, and then presents the related numerical and experimental results. Section III describes the configuration of stacked MSs with intriguing guiding and switching effects. Section IV includes a summary and conclusions of this work. Throughout the work, the timefactor $\exp (j \omega t)$, where $\omega$ is the angular frequency, and $t$ is the time, is assumed and suppressed.

\section{Configurations and Results - Single Metasurface}

Fig.1(a) shows the top (left) and the side (right) view of a single unit cell of the MS. It consists of a water element in a Rohacell $51 \mathrm{HF}$ host. The water element has the shape of a rounded rectangular cuboid; it comprises two half-cylinders of radius $r$ and length $d_{e}$ separated by a rectangular parallelepiped of width $r$. The height and thickness of the water 
element is denoted by $h$ and $d_{e}$, respectively. The width and height of the host are denoted by $a$ (this is the lattice constant of the resulting MS) and its thickness is $d$. A rectangular coordinate $\operatorname{system}(x, y, z)$ is shown and angle $\phi$ describes the rotation of the unit cell. All numerical investigations were performed in Comsol Multiphysics [13]; the dielectric function of water was taken from [14], and the in-house measured relative permittivity of 1.075 for Rohacell $51 \mathrm{HF}$ was used in simulations.

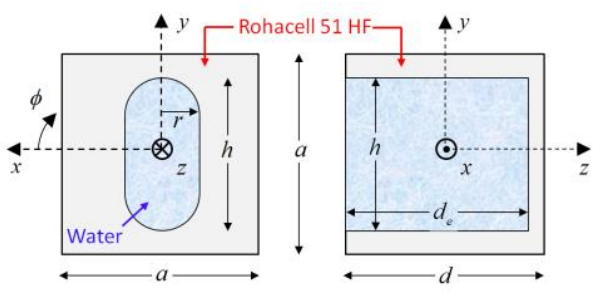

(a)

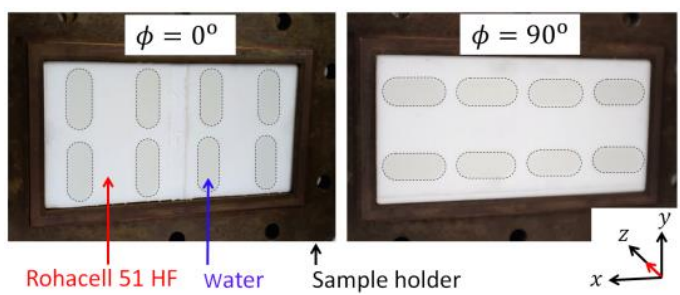

(b)

Fig. 1. The water-based unit cell (a) and fabricated MSs (b). Propagation is considered along the z-direction.

Characterization of the MSs was conducted in an L-band rectangular waveguide (WG-430). Its inner dimensions are 109.2 $\times 54.6 \mathrm{~mm}$, and its fundamental $\mathrm{TE}_{10}$ mode cut-off frequency range is $1.37-2.75 \mathrm{GHz}$. The number of unit cells in MSs ( 8 units) is defined by the size of the waveguide and the lattice constant. The elements are drilled, filled with water, and sealed with an adhesive tape; their parameters are: $a=27.3 \mathrm{~mm}, r=5 \mathrm{~mm}, h=22.5 \mathrm{~mm}, d_{e}=18.7 \mathrm{~mm}$ and $d=25 \mathrm{~mm}$. The cross-sections of the MSs are shown in Fig. 1(b). Their transmission and reflection were obtained using the HP 8753D Vector Network Analyzer. The red arrow in the $x y z$-coordinate system in Fig. 1(b) shows the incident wave propagation direction. The water element boundaries are indicated with dashed lines for the sake of clarity.

Mechanical tunability is characterized via changes in transmittance $\mathrm{T}$ and reflectance $\mathrm{R}$. The MS was optimized though extensive manual variations of its geometric parameters with the aim of suppressing transmitted fields for the $\phi=0^{\circ}$ orientation, while restoring the pass-band regime upon the $90^{\circ}$ rotation. Fig. 2 shows $\mathrm{T}$ and $\mathrm{R}$ as functions of frequency for (a) $\phi=0^{\circ}$ and (b) $\phi=90^{\circ}$. Numerical results are also included, and they display excellent agreement with experiments.

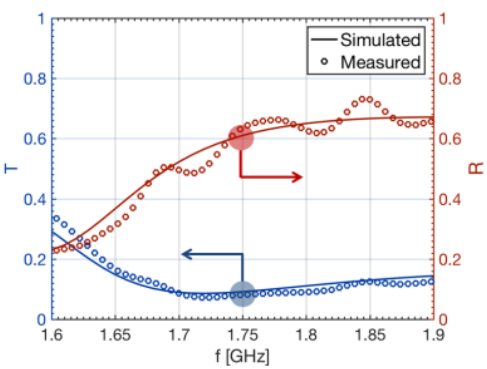

(a)

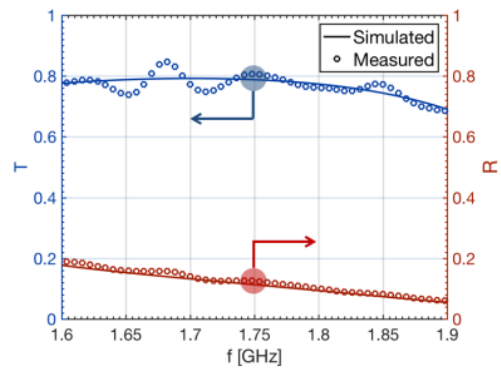

(b)

Fig. 2. Transmittance, T, and reflectance, R, as a function of frequency for two MS orientations $\phi=0^{\circ}$ (a) and $\phi=90^{\circ}$ (b).

For $\phi=0^{0}$ the measured transmittance minimum of $7 \%$ is at $1.72 \mathrm{GHz}$ where about $50 \%$ of the power is reflected (and rest $43 \%$ absorbed). Rotating the MS by $90^{\circ}$ changes the results such that now approximately $76 \%$ of the incident power is transmitted, while only $13 \%$ is reflected and $11 \%$ absorbed. Thus, mechanical rotation of the MS functions like a switch, changing the transmission by approximately $70 \%$. The difference between the two orientations of the MS can be understood in direct analogy with performance of a wire-grid (or plate-grid) polarizer exhibiting the on/off function for two

o the above Miss were optimized for $1.7 \mathrm{GHz}$ for the sake of simple and controllable characterization. Their performance can be improved at lower frequencies with the reduction of water losses. We scaled up (in size) the basic unit cell ( $a=$ $d=50 \mathrm{~mm}, r=10.7 \mathrm{~mm}, h=d_{e}=45 \mathrm{~mm}$ ) to obtain the transmittance minimum at $800 \mathrm{MHz}$. The MS was illuminated by a normally incident plane wave in free space. We used periodic boundary conditions in the numerical setup. The results are shown in Fig.3. As seen from Fig. 3(a), less than $1 \%$ of the incident power is transmitted, and $78 \%$ reflected in the $\phi=0^{\circ}$ orientation. Rotation by $90^{\circ}$ allows 93\% of the incident power be transmitted. Thus, quite effective switching is possible in the $0.8-0.9 \mathrm{GHz}$ range by rotating the MS. The observed behavior is explained by the excitation of the magnetic dipole resonances in the water elements. This is confirmed in Fig.3(b) which shows the normalized magnetic $(m)$ and electric $(e)$ polarizabilities, $\alpha_{m . e}^{n}=\alpha_{m . e}^{\prime}-j \alpha_{m . e}^{\prime \prime}$, obtained by the well-known formulas 
$[5,15]$. A magnetic dipole resonance is found close to the transmittance minimum at $800 \mathrm{MHz}$, Fig. 3(a). It is clear that the elements must have a certain electrical size in order to support such modes, and moreover, that any change of the geometry will deteriorate their excitation and thus the performance of the MSs.

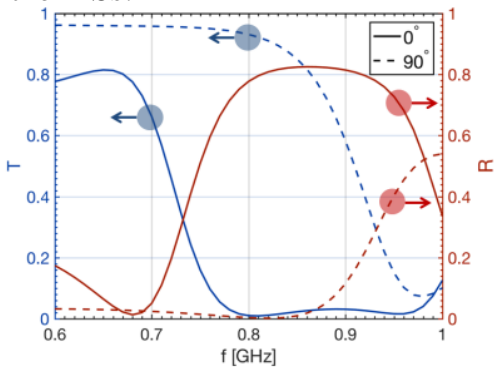

(a)

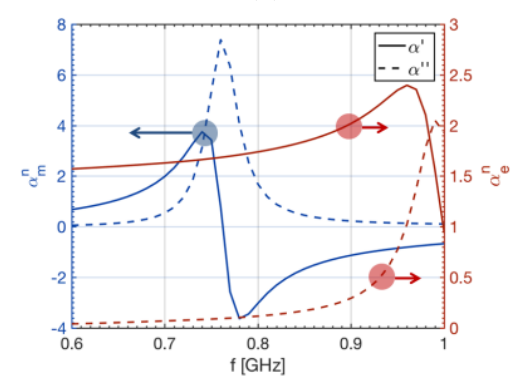

(b)

Fig. 3. Transmittance, $T$, and reflectance, $R$, as a function of frequency for the two MS orientations (a) and the resulting normalized magnetic and electric polarizabilities (b) for $\phi=0^{\circ}$. The water elements were optimized for $800 \mathrm{MHz}$.

While the MS characterizations were performed at a fixed temperature, the effects of thermal tunability can be easily accounted for in our numerical models. While the details of such a study will be reported elsewhere, we expect similar trend to apply presently as was found in [5] for spherical inclusions. As the temperature increases, the real part of the water permittivity decreases, thereby slightly blue-shifting the dipole resonances. Even though water losses increase slightly with increasing frequency, the effect of increase demperature leads to overall loss reduction, and thus to a stronger dipole excitation and more profound minima in the transmission coefficient. Exactly the opposite behavior takes place when the temperature is decreased.

Due to the nature of our experimental waveguide setup, the above results are valid for normal incidence only. The reported switching properties for single-layer MSs are expected to deteriorate for obliquely incident waves. A detailed study on the effects of varying angle of incidence is not included in here, but will be reported elsewhere.

\section{Multiple Metasurfaces - Waveguiding And SWITCHING EFFECTS}

Switching transmission on and off can be enhanced by stacking $N$ identical MSs as shown in Fig. 4. Each of the MSs has $3 \times 3$ unit cells. A rectangular coordinate $\operatorname{system}(x, y, z)$ is introduced such that its origin is in the center of the second MS and the MSs are stacked along the $z$-direction.

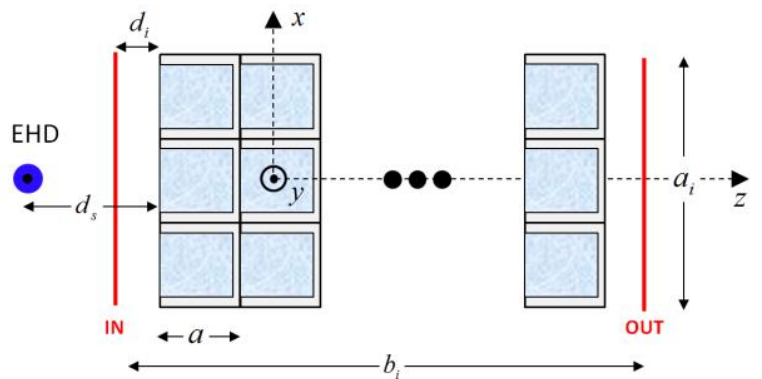

Fig. 4. The side view of the configuration of $N$ stacked MS excited by an electric Hertzian dipole.

We probe the stacked MSs with a $y$-directed electric Hertzian dipole (EHD) located at $d_{s}=50 \mathrm{~mm}$ from the first MS, see Fig. 4. The switching performance is quantified by the transmittance, T, given by $\mathrm{T}=\int_{\mathrm{OUT}} \boldsymbol{s} \cdot d \boldsymbol{s} / \int_{\mathrm{IN}} \boldsymbol{S}_{\mathrm{EHD}} \cdot d \boldsymbol{s}$, where $\boldsymbol{S}$ is the power flow density of the stacked MSs configuration, while $\boldsymbol{S}_{\mathrm{EHD}}$ is the power flow density of the EHD in free space. The integrations are performed over the quadratic surfaces IN and OUT parallel to the MSs; both have the side length of $3 a$. The IN surface is located at distance $d_{i}=a / 4=12.5 \mathrm{~mm}$ before the first MS, and the OUT surface is located at distance $b_{i}=(N+1 / 2) a$ after the IN surface (Fig. 4). In all cases, the dipole moment amplitude is set to $0.001 \mathrm{Am}$.

The $90^{\circ}$ orientation of the unit cells guides the transmitted power; this is termed as the ON state and the corresponding transmittance is Ton. We expect that the transmitted power is effectively blocked in the $0^{\circ}$ orientation of the MSs; this is termed the OFF state with transmittance $T_{\mathrm{OFF}}$. The guiding effect is illustrated in Fig.5 (a). TON and TofF are shown as functions of $N$. The reference transmittance, $\mathrm{T}_{\mathrm{EHD}}$, obtained for $\boldsymbol{S}=\boldsymbol{S}_{\text {EHD }}$, decreases, as expected, notably with increasing $N$. The transmittance of the stacked MS configuration, ToN, also decreases due to losses but not as fast as the reference transmittance. Fig.5 (b) depicts the guiding efficiency calculated as $\eta=\mathrm{T}_{\mathrm{ON}} / \mathrm{T}_{\mathrm{EHD}}$ (in \%), and it is found of growing rapidly with increasing $N$. In particular, for $N=7$, the stacked MS configuration concentrates and guides (in the $z$-direction) 10 times more power than free space.

The switching effect is also illustrated in Fig. 5. Obviously, the transmitted power is very low in the OFF state. Fig. 5 (b) depicts the switching efficiency calculated as $\rho=\mathrm{T}_{\mathrm{ON}} / \mathrm{ToFF}$; it increases rapidly with increasing $N$. In particular, for $N=7, \rho>$ 1400 clearly quantifying the enhancement of the switching effect by stacking MSs. The switching effect is nicely visualized by the plots of the power flow densities for the ON and OFF states with $N=7$ shown in the $y=0$ plane in Fig. 5(c) and (d), respectively. The colors depict the magnitude of the power flow density $\left(10 \log _{10}\left|S / 1\left[\mathrm{~W} / \mathrm{m}^{2}\right]\right|\right)$, while the arrows show its direction. In the ON state (Fig. 5(c)), the power density is uniformly concentrated in the direction of the alignment with very large output values. In the OFF state (Fig. $5(d)$ ), the power flow density is effectively blocked by the 
stack. This means that the sufficient switching effect may require a fewer number of MSs than 7.

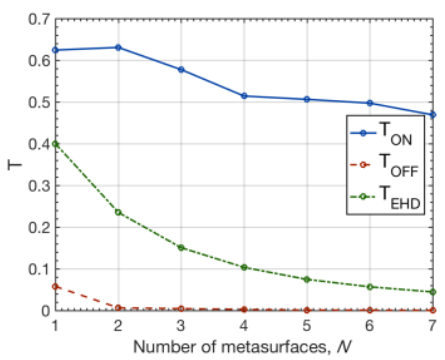

(a)

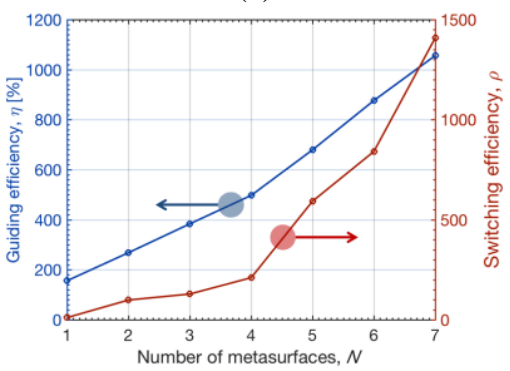

(b)

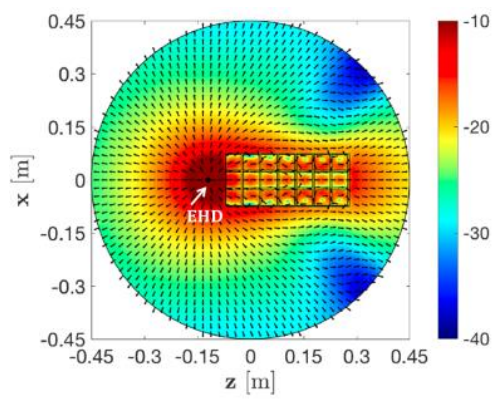

(c)

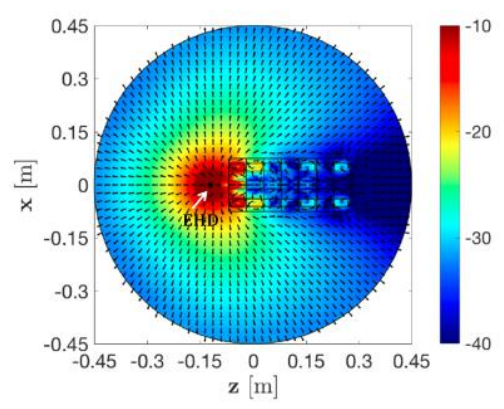

(d)

Fig. 5. (a) Transmittances $\mathrm{T}_{\mathrm{ON}}, \mathrm{T}_{\mathrm{OFF}}$ and $\mathrm{T}_{\mathrm{EHD}}$ ( (b) Guiding efficiency $\eta$ and switching efficiency $\rho$. All results are shown as a function of the number of MSs, $N$. The power flow density in the $x z$-plane $(y=$ 0 ) for $N=7 \mathrm{MSs}$ in ON (c) and (d) OFF states. The color (arrows) shows the magnitude (direction) of the power flow density.

\section{SuMmary AND CONCluSIONS}

Mechanically tunable MSs utilizing simple "rod-like" water inclusions were designed. Due to the polarization sensitivity of the inclusions, their transmission and reflection properties can be easily tailored from low to high values through mechanical rotation of the MSs by $90^{\circ}$. Performance of the individual MSs was characterized experimentally in an L-band rectangular waveguide, and excellent agreement with numerical results was found. Moreover, it was shown numerically that a stack of MSs in free space can guide waves of a near-by dipole antenna. This radiation can be turned on and off through rotations of the entire stack of MSs by $90^{\circ}$, thus indicating their potential in the design of simple, cheap and bio-friendly tunable microwave components. We admit that the waterbased MSs cannot compete with other tunable and switchable microwave devices in terms of speed or modulation frequency. However, the main advantage of the proposed structures is its extremely low cost, abundance and ecological compatibility. In addition, since the sources of microwave radiation are inexpensive, this provides not only a way of quick and cheap prototyping of MSs, but also their possible applications for educational and science-popularization purposes.

While the present work focused on mechanical tunability of water-based MSs, our future efforts will account for their reconfigurability aspects. This can be accomplished through e.g., thermal tunability enabled by a strong temperature dependence of the water permittivity [5, 14]. Moreover, by letting the inclusions be partially filled with water, as reported in $[5,8]$, even small rotations result in very tunable responses as water, strictly following the shape of the MS inclusions.

\section{REFERENCES}

[1] S. Jahani and Z. Jacob, "All-dielectric metamaterials," Nat. Nanotechnol. 11, 23-36, 2016.

[2] A. I. Kuznetsov, A. E. Miroshnichenko, M. Brongersma, Y.S. Kivshar, and B.S. Luk'yanchuk, "Optically resonant dielectric nanostructures", Science 354, 2472, 2016.

[3] I. Staude and J. Schilling,"Metamaterial-inspired silicon nanophotonics", Nature Photonics 11, 274-284, 2017.

[4] E. N. Bunting, G. R. Shelton, and A. S. Creamer, "Properties of bariumstrontium titanate dielectrics," J. Am. Ceramic So. 30, 114-125, 1947.

[5] A. Andryieuski, S. M. Kuznetsova, S. V. Zhukovsky, Y. S. Kivshar, and A. V. Lavrinenko, "Water: promising opportunities for tunable alldielectric electromagnetic metamaterials," Sci. Rep. 5, 13535, 2015.

[6] Y. J. Yoo et al. "Metamaterial absorber for electromagnetic waves in periodic water droplets," Sci. Rep. 5, 14018, 2015.

[7] Q. Song et al. "Water-resonator-based metasurface: an ultrabroadband and near-unity absorption," Adv. Opt. Mat. 5, 1601103, 2017.

[8] M. Odit, P. Kapitanova, A. Andryieuski, P. Belov and A. V. Lavrinenko,"Experimental demonstration of water based tunable metasurface," Appl. Phys. Lett. 109, 011901, 2016.

[9] M. A. Gorlach, M. Song, A. P. Slobozhanyuk, A. A. Bogdanov and P. Belov, "Topological transition in coated wire medium," Phys. Status Solidi RRL 1-5, 2016.

[10] Y. Pang et al."Thermally tunable water-substrate broadband metamaterial absorbers," Appl. Phys. Lett. 110, 104103, 2017.

[11] P. Kapitanova, V. Ternovski, A. Miroshnichenko, N. Pavlov, P. Belov, Y. Kivshar, and M. Tribelsky, "Giant field enhancement in high-index dielectric subwavelength particles," Sci. Rep. 7, 731, 2017.

[12] X. Yang, D Zhang, S. Wu, L. Li, K. Cao, and K. Huang, "Reconfigurable all-dielectric metasurface based on tunable chemical systems in aqueous solution," Sci. Rep. 7, 3190, 2017.

[13] COMSOL Multiphysics 5.2a; https://www.comsol.com/

[14] W. Ellison, "Permittivity of pure water, at standard atmospheric pressure, over the frequency range $0-25$ thz and the temperature range 0-100 c. J. Phys. Chem. Ref. Data 36, 1-18, 2007.

[15] S. Tretyakov Analytical modeling in applied electromagnetics, Artech House, 2003.

[16] B. A. Munk, Frequency selective surfaces, John Wiley \& Sons, 2000. 